\title{
Is 'planning' missing from our family planning services?
}

\author{
Sarah Crede, Jane Harries, Deborah Constant, Theresa Hatzell Hoke, Mackenzie Green, Jennifer Moodley
}

To the Editor: The role of family planning in achieving the Millennium Development Goals is well recognised. ${ }^{1}$ The benefits of family planning, in developing countries in particular, extend beyond decreasing fertility and include poverty reduction, improved health for both mother and child, the promotion of gender equality by increasing women's opportunities beyond reproductive and domestic activities, and environmental sustainability. ${ }^{1}$ In addition, prevention of undesired pregnancies among HIV-positive women by eliminating unmet need for contraception is a highly cost-effective means of preventing mother-to-child transmission. ${ }^{2}$

In South Africa, free contraceptives are available at public sector health care facilities, and contraception use is high: an estimated $65 \%$ of sexually active women use a method. ${ }^{3}$ The method mix comprises predominantly short-acting methods - primarily injectable contraceptives. Long-acting contraceptives, such as the intra-uterine device (IUD), are highly effective among typical users owing to consistency of function, yet are underutilised in South Africa's public sector facilities. Of importance, especially in South Africa's high HIVprevalent setting, is that the IUD can be safely used on clinically well HIV-positive women. ${ }^{4}$ The 2004 Demographic and Health Survey showed that $10 \%$ of sexually active women were sterilised, while less than $1 \%$ of women were using the IUD. ${ }^{3}$ In preparation for an intervention aimed at improving contraceptive options, including long-acting and permanent methods (LAPM), for all postpartum women, we assessed women's knowledge and attitudes to LAPM. We report on findings from our baseline survey, which have prompted the question: Where is the 'planning' in family planning services?

\section{Methods}

A cross-sectional study, approved by the University of Cape Town Health Science Faculty Human Research Ethics Committee, was conducted at five public sector primary care facilities in Cape Town between February and April 2009. Trained interviewers using a structured questionnaire collected data on: socio-demographics, contraceptive use, future fertility desires, and knowledge and attitudes towards LAPM, from 538 women of whom 265 were HIV-positive and 273 HIV-negative. The sample included HIV-positive and negative women to enable a comparison to be made of the uptake of an LAPM promotion intervention between prevention of mother-

Women's Health Research Unit, School of Public Health and Family Medicine, University of Cape Town

Sarah Crede, BSc (Physiotherapy)

Jane Harries, BA (Hons), MPhil, MPH

Deborah Constant, BSc (Physiotherapy), BSc (Hons), MSc Med, MPH

Jennifer Moodley, MB ChB, MMed (Public Health)

Family Health International, North Carolina, USA

Theresa Hatzell Hoke, MPH, PhD (Health Policy and Administration)

Mackenzie Green, MHS to-child transmission (PMTCT) and non-PMTCT clients. All study participants were attending child health services, had received antenatal care during their most recent pregnancy, and had delivered less than 6 months before the interview.

\section{Results}

The median age of the women was 26 years (interquartile range (IQR) 22 - 31), 63.9\% had achieved Grade 8 - 12 without matric, 29.5\% had post-matric qualifications, and the vast majority $(94.2 \%)$ reported to be either married or single but in a stable relationship. Participants had a median number of 2 (IQR 1 - 2) living children. Overall, 67.3\% of the women did not want another child in the future, $39 \%$ reported that their partners did not want another child, and $62.4 \%$ reported that their most recent pregnancy was unplanned. Of the $19.3 \%$ of women who wanted another child in the future, $52 \%$ wanted to wait more than 36 months before doing so.

Current use of a method of contraception was reported by $89 \%$, namely: injection $(90.2 \%)$, sterilisation $(6.7 \%)$, condom $(4.4 \%)$ and the pill (1.7\%), but none was using an IUD.

Using a knowledge score comprising 6 questions (maximum score 6), current knowledge about the IUD was found to be low (median 2 and range 0 - 4). Knowledge about sterilisation was higher: maximum score of 5 , median 3 (range 2 - 4).

Since the birth of their youngest child, $53.5 \%$ reported that a health care provider had spoken to them about family planning methods to avoid pregnancy. Of those who had discussed family planning methods with a health care provider, only $9 \%$ reported that the health care provider had talked about the IUD as a family planning method, while $51.4 \%$ reported that sterilisation had been discussed.

\section{Discussion}

The finding that $67.3 \%$ of women did not want another child in the future, and that more than half of those who wanted another child, wanted to wait at least 3 years before doing so, suggests the need for long-acting contraceptives in this community. In our study, there was a serious mismatch between the fertility desires of the participants and their current use of short-term contraceptive methods. No women were using the IUD, which is more than $98 \%$ effective in preventing pregnancy ${ }^{5}$ and offers a quick return to fertility after removal. During data collection, none of the nurses at the five study sites was trained in IUD insertion and removal, and it is therefore not surprising that IUDs are not being used in family planning. As this service is not available in these study settings, or in many clinics in the Western Cape, its promotion is unlikely.

Although the high rate of unplanned pregnancies in our study is not a new finding, it is cause for concern. We found a higher rate of use of short-acting methods than has been nationally reported. ${ }^{3}$ Shortacting methods, such as injectable contraceptives, are highly effective forms of contraception when used consistently. However, they require women to visit health care facilities at regular 2 - 3-monthly intervals, placing an increased burden on clinic staff and allowing opportunity for inconsistent use and subsequent unintended pregnancies. A study in the Western and Eastern Cape provinces found that a large proportion of women who intended to continue using the injection were late for their scheduled re-injection, and in some cases beyond the 'grace period' during which re-injection can still be administered. ${ }^{6}$ The high rate of unplanned pregnancies reported in this study may therefore be partially explained by inconsistent or discontinued use of 
short-acting contraceptive methods, with inadequate counselling and barriers in the health care system being contributory factors in such contraceptive failure.

Participant knowledge of sterilisation was greater than that regarding IUDs in this study, probably owing to the presence of sterilisation services in the Western Cape and the lack of IUD promotion and use. The Association for Voluntary Sterilization South Africa (AVSSA), a non-profit organisation, has offered sterilisation services at health care facilities in the Western Cape for many years, including education and awareness campaigns.

The survey results raise questions as to whether the full range of contraceptive methods are available and promoted in all services targeting pregnant and postpartum women, including HIV-positive women. They also suggest that recommended education and counselling regarding healthy timing and spacing of children may be overlooked in health services serving pregnant and postpartum women. High rates of unplanned pregnancy are likely to continue without effective planning between a woman and her partner regarding family size, spacing of children and the selection of wellsuited contraceptive methods that are supported by family planning service providers at health care facilities.

Choice is essential in providing the optimal family planning method to a client. Women in the communities studied have insufficient knowledge about safe, effective and long-acting contraceptive options and therefore are unable to make a fully informed method choice. To provide family planning clients with a choice of contraceptive methods, providers must be adequately trained and equipped to provide counsel about, and offer the full range of, methods available in the public health care sector. Family planning should be appropriate and well-suited to the individual's health and fertility desires. Given their superior effectiveness rates among typical users, long-acting and permanent methods are excellent options for clients who have achieved their desired family size or for those who have recently given birth and wish to plan future pregnancies, and such methods should therefore be promoted and available to clients as part of comprehensive family planning services.

\section{References}

1. Cleland J, Bernstein S, Ezeh A, Faundes A, Glasier A, Innis J. Family planning: the unfinished agenda, Lancet 2006; 368: 1810-1827.

2. Halperin DT, Stover J, Reynolds H. Benefits and costs of expanding access to family planning programs to women living with HIV. AIDS 2009; 23 (suppl 1): S123-S130.

3. Department of Health. Demographic and Health Survery 2003, Preliminary Report. Pretoria: Department of Health, 2004. http://www.doh.gov.za (accessed 20 January 2010).

4. World Health Organization. Improving Access to Quality Care in Family Planning: Medical Eligibility Criteria for Contraceptive Use. 3rd ed. Geneva: World Health Organization, 2004

5. Thonneau P, Almont T. Contraceptive efficacy of intrauterine devices. Am J Obstet Gynecol 2008; 198(3): 248-253.

6. Baumgartner JN, Morroni C, Mlobeli RD, et al. Timeliness of contraceptive reinjections in South Africa and its relation to unintentional discontinuation. Int Fam Plan Perspect 2007; 33(2): 66-74.

Accepted 3 March 2010

\title{
New fly species causing human myiasis identified in Eastern Cape, South Africa
}

\author{
S K Kuria, H J C Kingu, S D Vasaikar, J N Mkhize, J M Iisa, A Dhaffala
}

To the Editor: Myiasis is the infestation of tissues of live vertebrates (humans and/or animals) by dipterous larvae. Human myiasis is classified according to the type of larva producing the lesion, location

Department of Biological Sciences, Walter Sisulu University, Mthatha, E Cape $S$ K Kuria, $\mathrm{PhD}$

J N Mkhize, PhD

J M Iisa, BVetMed

Department of Surgery, Walter Sisulu University

H J C Kingu, MD, MMed (Surg)

A Dhaffala, MB ChB, MMed (Surg)

Department of Medical Microbiology, Walter Sisulu University

S D Vasaikar, MD (Med Microbiol), MB BS and clinical signs. ${ }^{1}$ Myiases in humans are thought to have originated from the close association between humans and domestic animals in ancient times. ${ }^{2}$ Flies that cause myiasis are grouped into three families: Calliphoridae (blowflies), Sarcophagidae (flesh flies) and Oestridae (bot flies). ${ }^{2}$ For a long period species of Sarcophaga have been implicated in myiasis in man and animals. ${ }^{3}$ Other dipterans have also been implicated with causing myiasis in humans, including Cochliomyia hominivorax (Coquerel), ${ }^{1}$ C. macellaria Fab., Phormia regina Meig., Lucilia sericata Meig., L. illustris Meig., Calliphora erythrocephala Meig. and Cynomyia cadaverina Desv. ${ }^{4}$ Nevertheless, several species of flies have been used for maggot debridement therapy (MDT), the commonest being L. sericata Meigen, a greenbottle blowfly which is closely related to the greenbottle $L$. cuprina Wiedmann; however, $L$. cuprina feeds on live as well as necrotic tissue, which is undesirable in MDT. ${ }^{5}$

We aimed to elucidate the fly species that cause myiasis in humans in the Eastern Cape province, South Africa. We collected maggots from necrotic human wounds in patients attending Nelson Mandela Academic Hospital (NMAH) and other clinics within the O R Tambo municipality. These maggots were allowed to grow and pupate, and the emerging flies were subsequently identified. ${ }^{6}$ 